\title{
SEMIGROUPS OF COMPOSITION OPERATORS IN BMOA AND THE EXTENSION OF A THEOREM OF SARASON
}

\author{
O. BLASCO, M.D. CONTRERAS, S. DÍAZ-MADRIGAL, J. MARTÍNEZ, AND A.G. SISKAKIS
}

\begin{abstract}
In this paper we deal with the maximal subspace in $B M O A$ where a general semigroup of analytic functions on the unit disk generates a strongly continuous semigroup of composition operators. Particular cases of this question are related to a well-known theorem of Sarason about $V M O A$. Our results describe analytically that maximal subspace and provide a condition which is sufficient for the maximal subspace to be exactly $V M O A$. A related necessary condition is also proved in the case when the semigroup has an inner Denjoy-Wolff point. As byproduct we provide a generalization of the theorem of Sarason.
\end{abstract}

\section{Introduction}

Let $\mathcal{H}(\mathbb{D})$ be the Fréchet space of all analytic functions in the unit disk endowed with the topology of uniform convergence on compact subsets of $\mathbb{D}$.

A (one-parameter) semigroup of analytic functions is any continuous homomorphism $\Phi: t \mapsto$ $\Phi(t)=\varphi_{t}$ from the additive semigroup of nonnegative real numbers into the composition semigroup of all analytic functions which map $\mathbb{D}$ into $\mathbb{D}$. In other words, $\Phi=\left(\varphi_{t}\right)$ consists of analytic functions on $\mathbb{D}$ with $\varphi_{t}(\mathbb{D}) \subset \mathbb{D}$ and for which the following three conditions hold:

(1) $\varphi_{0}$ is the identity in $\mathbb{D}$,

(2) $\varphi_{t+s}=\varphi_{t} \circ \varphi_{s}$, for all $t, s \geq 0$,

(3) $\varphi_{t} \rightarrow \varphi_{0}$, as $t \rightarrow 0$, uniformly on compact subsets of $\mathbb{D}$.

It is well known that condition (3) above can be replaced by

$\left(3^{\prime}\right)$ For each $z \in \mathbb{D}, \varphi_{t}(z) \rightarrow z$, as $t \rightarrow 0$.

Each such semigroup gives rise to a semigroup $\left(C_{t}\right)$ consisting of composition operators on $\mathcal{H}(\mathbb{D})$,

$$
C_{t}(f):=f \circ \varphi_{t}, \quad f \in \mathcal{H}(\mathbb{D}) .
$$

We are going to be interested in the restriction of $\left(C_{t}\right)$ to certain linear subspaces $\mathcal{H}(\mathbb{D})$. Given a Banach space $X$ consisting of functions of $\mathcal{H}(\mathbb{D})$ and a semigroup $\left(\varphi_{t}\right)$, we say that $\left(\varphi_{t}\right)$ generates a semigroup of operators on $X$ if $\left(C_{t}\right)$ is a well-defined strongly continuous semigroup of bounded operators in $X$. This exactly means that for every $f \in X$, we have $C_{t}(f) \in X$ for all $t \geq 0$ and

$$
\lim _{t \rightarrow 0^{+}}\left\|C_{t}(f)-f\right\|_{X}=0 .
$$

Thus the crucial step to show that $\left(\varphi_{t}\right)$ generates a semigroup of operators in $X$ is to pass from the pointwise convergence $\lim _{t \rightarrow 0^{+}} f \circ \varphi_{t}(z)=f(z)$ on $\mathbb{D}$ to the convergence in the norm of $X$.

This connection between composition operators and semigroups opens the possibility of studying spectral properties, operator ideal properties or dynamical properties of the semigroup of operators $\left(C_{t}\right)$ in terms of the theory of functions. Papers [1] and [10] can be considered as the starting points in this direction.

Classical choices of $X$ treated in the literature are the Hardy spaces $H^{p}$, the disk algebra $A(\mathbb{D})$, the Bergman spaces $A^{p}$, the Dirichlet space $\mathcal{D}$ and the chain of spaces $Q_{p}$ and $Q_{p, 0}$ which have

Date: July 28, 2006.

2000 Mathematics Subject Classification. Primary 30H05, 32A37, 47B33, 47D06; Secondary 46E15.

Key words and phrases. Semigroups, composition operators, BMOA, VMOA.

This research has been partially supported by the Ministerio de Ciencia y Tecnología projects n. BFM200307294-C02-02 and MTM2005-08350-C03-03 and by La Consejería de Educación y Ciencia de la Junta de Andalucía. 
been introduced recently and which include the spaces BMOA, Bloch as well as their "little oh" analogues. See [19] and [20]for definitions and basic facts of the spaces and [15], [16], and [18] for composition semigroups on these spaces.

Very briefly, the state of the art is the following: (i) Every semigroup of analytic functions generates a semigroup of operators on the Hardy spaces $H^{p}(1 \leq p<\infty)$, the Bergman spaces $A^{p}$ $(1 \leq p<\infty)$, the Dirichlet space, and on the spaces VMOA and little Bloch. (ii) No non-trivial semigroup generates a semigroup of operators in the space $H^{\infty}$ of bounded analytic functions. (iii) There are plenty of semigroups (but not all) which generate semigroups of operators in the disk algebra. Indeed, they can be well characterized in several analytical terms [4].

In this paper we concentrate on the space $B M O A$. As we will see, the strong continuity behavior differs notably from other known cases, since it depends heavily on the particular semigroup.

This has led us to introduce the following notation: Given a semigroup $\left(\varphi_{t}\right)$ we denote by $\left[\varphi_{t}, B M O A\right]$ the maximal closed linear subspace of $B M O A$ such that $\left(\varphi_{t}\right)$ generates a semigroups of operators on it. The existence of such a maximal subspace, as well as analytical descriptions of it will be discussed in section two. In that section, we also present an alternative self-contained proof of the fact that every semigroup generates a semigroup of operators on $V M O A$. This in particular means that in our notation

$$
V M O A \subseteq\left[\varphi_{t}, B M O A\right]
$$

for every semigroup $\left(\varphi_{t}\right)$. It is important to underline that, in general, $V M O A \mp\left[\varphi_{t}, B M O A\right]$.

The chain of inclusions $V M O A \subseteq\left[\varphi_{t}, B M O A\right] \subseteq B M O A$ leads us to wonder about those semigroups with an extreme character, that is, those giving equality

$$
V M O A=\left[\varphi_{t}, B M O A\right] \quad \text { or } \quad\left[\varphi_{t}, B M O A\right]=B M O A .
$$

In section three we deal with the left hand equality $V M O A=\left[\varphi_{t}, B M O A\right]$, and present a sufficient condition on the semigroup for this equality to hold. A different but closely related condition is shown to be necessary for semigroups with inner Denjoy-Wolff point. The conditions are in terms of the growth of the infinitesimal generator of $\left(\varphi_{t}\right)$ near the boundary of $\mathbb{D}$.

There is an important connection of the above results with a well-known theorem of D. Sarason which characterizes the space VMOA. Namely, Sarason [12], [13] proved

Theorem A. (Sarason [12]) Suppose $f \in B M O A$, then the following are equivalent:

(1) $f \in V M O A$.

(2) $\lim _{t \rightarrow 0^{+}}\left\|f\left(e^{i t} z\right)-f\right\|_{B M O A}=0$.

(3) $\lim _{t \rightarrow 0^{+}}\left\|f\left(e^{-t} z\right)-f\right\|_{B M O A}=0$.

In our notation this theorem can be written as

$$
V M O A=\left[e^{i t} z, B M O A\right]=\left[e^{-t} z, B M O A\right] .
$$

In other words, the semigroups $\varphi_{t}(z)=e^{i t} z$ of rotations and $\varphi_{t}(z)=e^{-t} z$ of dilatations with respect to the origin are left-extreme in the above sense. Our results provide many more nontrivial examples of semigroups of this type.

We end this introduction by presenting a quick review of basic facts about a general semigroup of analytic functions (see [16]). The basic material about operator semigroups on Banach spaces can be found in $[7$, Chapter VIII].

If $\left(\varphi_{t}\right)$ is a semigroup, then each map $\varphi_{t}$ is univalent. The infinitesimal generator of $\left(\varphi_{t}\right)$ is the function

$$
G(z):=\lim _{t \rightarrow 0^{+}} \frac{\varphi_{t}(z)-z}{t}, z \in \mathbb{D} .
$$

This convergence holds uniformly on compact subsets of $\mathbb{D}$ so $G \in \mathcal{H}(\mathbb{D})$. Moreover $G$ satisfies

$$
G\left(\varphi_{t}(z)\right)=\frac{\partial \varphi_{t}(z)}{\partial t}=G(z) \frac{\partial \varphi_{t}(z)}{\partial z}, z \in \mathbb{D}, t \geq 0 .
$$


Further $G$ has a unique representation

$$
G(z)=(\bar{b} z-1)(z-b) P(z), z \in \mathbb{D}
$$

where $b \in \overline{\mathbb{D}}$ and $P \in \mathcal{H}(\mathbb{D})$ with $\operatorname{Re} P(z) \geq 0$ for all $z \in \mathbb{D}$. If $G$ is not identically null, the couple $(b, P)$ is uniquely determined from $\left(\varphi_{t}\right)$ and the point $b$ is called the Denjoy-Wolff point of the semigroup. We want to mention that this point plays a crucial role in the dynamical behavior of the semigroup (see [16], [5]).

Note that for $r$ sufficiently near to one, it is clear from the above representation of $G$ that $G$ has no zeros in the annulus $r<|z|<1$, so $1 / G$ is analytic on that annulus. This remark will be implicitly used throughout the paper.

\section{Semigroups in BMOA}

For the sake of completeness and to fix notations, we begin with a quick review of basic properties of $V M O A$ and $B M O A$.

$B M O A$ is the Banach space of all analytic functions in the Hardy space $H^{2}$ whose boundary values have bounded mean oscillation. There are many characterizations of this space but we will use the one in terms of Carleson measures (see [20,9]). Namely, a function $f \in H^{2}$ belongs to $B M O A$ if and only if there exists a constant $C>0$ such that

$$
\int_{R(I)}\left|f^{\prime}(z)\right|^{2}\left(1-|z|^{2}\right) d A(z) \leq C|I|
$$

for any arc $I \subset \partial \mathbb{D}$, where $R(I)$ is the Carleson rectangle determined by $I$, that is,

$$
R(I):=\left\{r e^{i \theta} \in \mathbb{D}: 1-\frac{|I|}{2 \pi}<r<1 \text { and } e^{i \theta} \in I\right\} .
$$

As usual, $|I|$ denotes the length of $I$ and $d A(z)$ the normalized Lebesgue measure on $\partial \mathbb{D}$. The corresponding $B M O A$ norm is

$$
\|f\|_{B M O A}:=|f(0)|+\sup _{I \subset \partial \mathbb{D}}\left(\frac{1}{|I|} \int_{R(I)}\left|f^{\prime}(z)\right|^{2}\left(1-|z|^{2}\right) d A(z)\right)^{1 / 2} .
$$

Trivially, each polynomial belongs to $B M O A$. The closure of all polynomials in $B M O A$ is denoted by $V M O A$. Alternatively, $V M O A$ is the subspace of $B M O A$ formed by those $f \in B M O A$ such that

$$
\lim _{|I| \rightarrow 0} \frac{1}{|I|} \int_{R(I)}\left|f^{\prime}(z)\right|^{2}\left(1-|z|^{2}\right) d A(z)=0 .
$$

Particular and quite interesting examples of members of $V M O A$ are provided by any function in the Dirichlet space $\mathcal{D}$, which is the space of those functions $f \in \mathcal{H}(\mathbb{D})$ such that $f^{\prime} \in L^{2}(\mathbb{D}, d A)$. In fact, for every $f \in \mathcal{D}$,

$$
\frac{1}{|I|} \int_{R(I)}\left|f^{\prime}(z)\right|^{2}\left(1-|z|^{2}\right) d A(z) \leq 2 \int_{R(I)}\left|f^{\prime}(z)\right|^{2} d A(z) \rightarrow 0 \text { as }|I| \rightarrow 0 .
$$

For more information on these Banach spaces, we refer the reader to the excellent monographs or [9] or [20].

In our first result, we confirm the existence of a maximal closed linear subspace of $B M O A$ on which a semigroup $\left(\varphi_{t}\right)$ generates a semigroup of operators. In this context, we recall that any analytic self map $\varphi$ of the disk induces a bounded composition operator $C_{\varphi}(f)=f \circ \varphi$ on $B M O A$ and there is a constant $C>0$, not depending on $\varphi$, such that

$$
\left\|C_{\varphi}\right\|_{B M O A} \leq C\left(1+\log \frac{1}{1-|\varphi(0)|}\right) .
$$

Moreover $C_{\varphi}$ is bounded on $V M O A$ if and only if $\varphi \in V M O A$, see [3] for details. 
Proposition 2.1. Let $\left(\varphi_{t}\right)$ be a semigroup of analytic functions. Then there exists a closed subspace $Y$ of BMOA such that $\left(\varphi_{t}\right)$ generates a semigroup of operators on $Y$ and such that any other subspace of BMOA with this property is contained in $Y$.

Proof. Consider the linear subspace of $B M O A$ defined by

$$
Y:=\left\{f \in B M O A: \lim _{t \rightarrow 0^{+}}\left\|f \circ \varphi_{t}-f\right\|_{B M O A}=0\right\} .
$$

Notice that $\sup _{t \in[0,1]}\left|\varphi_{t}(0)\right|=M<1$. Hence from (2.2),

$$
\sup _{t \in[0,1]}\left\|C_{t}\right\|_{B M O A} \leq C(1-\log (1-M))<+\infty .
$$

This and the triangle inequality for norms shows that $Y$ is a closed subspace of $B M O A$. Thus in order to prove that $\left(\varphi_{t}\right)$ generates a semigroup of operators in $Y$, it remains to check that if $f \in Y$ then $C_{s}(f) \in Y$ for all $s \geq 0$. To see this let $s, t \geq 0$, then

$$
\left\|C_{s}(f) \circ \varphi_{t}-C_{s}(f)\right\|_{B M O A} \leq C\left(1+\log \frac{1}{1-\left|\varphi_{s}(0)\right|}\right)\left\|f \circ \varphi_{t}-f\right\|_{B M O A} \rightarrow 0 \text { as } t \rightarrow 0^{+} .
$$

Finally, if $W$ is a subspace of $B M O A$ such that $\left(\varphi_{t}\right)$ generates a semigroup of operators on $W$, then for any $f \in W$ we have in particular $\lim _{t \rightarrow 0^{+}}\left\|f \circ \varphi_{t}-f\right\|_{B M O A}=0$, thus $f \in Y$ and we conclude $W \subset Y$.

In what follows, this maximal subspace $Y$ will be denoted as $\left[\varphi_{t}, B M O A\right]$. It is easy to see that if $Z$ is any closed subspace of $\left[\varphi_{t}, B M O A\right]$ which is invariant under $\left(C_{t}\right)$ (i.e. $C_{t}(Z) \subset Z$ for every $t \geq 0)$ then $\left(\varphi_{t}\right)$ generates a semigroups of operators on $Z$.

This maximal subspace can be also described directly in terms of the infinitesimal generator.

Theorem 2.2. Let $G$ be the infinitesimal generator of $\left(\varphi_{t}\right)$. Then,

$$
\left[\varphi_{t}, B M O A\right]=\overline{\left\{f \in B M O A: G f^{\prime} \in B M O A\right\}} .
$$

Proof. We may assume that $\left(\varphi_{t}\right)$ is not trivial. Denote by $\Gamma$ the infinitesimal generator of the operator semigroup $\left(C_{t}\right)$ acting on the Banach space $\left[\varphi_{t}, B M O A\right]$, and by $\mathcal{D}(\Gamma)$ its domain. We will show that if $f \in \mathcal{D}(\Gamma)$ then $G f^{\prime} \in B M O A$. Indeed if $f \in \mathcal{D}(\Gamma)$ then $\Gamma(f) \in B M O A$ and

$$
\lim _{t \rightarrow 0^{+}}\left\|\frac{1}{t}\left(C_{t}(f)-f\right)-\Gamma(f)\right\|_{B M O A}=0 .
$$

Since convergence in the $B M O A$ norm implies uniform convergence on compact subsets of $\mathbb{D}$ and therefore in particular pointwise convergence, for each $z \in \mathbb{D}$ we have

$$
\begin{aligned}
\Gamma(f)(z) & =\lim _{t \rightarrow 0^{+}} \frac{f\left(\varphi_{t}(z)\right)-f(z)}{t}=\lim _{t \rightarrow 0^{+}} \frac{f\left(\varphi_{t}(z)\right)-f\left(\varphi_{0}(z)\right)}{t} \\
& =\left.\frac{\partial f \circ \varphi_{t}(z)}{\partial t}\right|_{t=0}=\left.f^{\prime}\left(\varphi_{0}(z)\right) \frac{\partial \varphi_{t}(z)}{\partial t}\right|_{t=0}=f^{\prime}(z) G(z),
\end{aligned}
$$

that is, $G f^{\prime}=\Gamma(f) \in B M O A$, and thus $\mathcal{D}(\Gamma) \subset\left\{f \in B M O A: G f^{\prime} \in B M O A\right\}$. Taking closures and bearing in mind the fact from the general theory of operator semigroups that $\mathcal{D}(\Gamma)$ is dense in $\left[\varphi_{t}, B M O A\right]$ we conclude

$$
\left[\varphi_{t}, B M O A\right] \subseteq \overline{\left\{f \in B M O A: G f^{\prime} \in B M O A\right\}} .
$$

Conversely, let $f \in B M O A$ such that $m:=G f^{\prime} \in B M O A$. First of all, we assert that

$$
\left(f \circ \varphi_{t}\right)^{\prime}(z)-f^{\prime}(z)=\int_{0}^{t}\left(m \circ \varphi_{s}\right)^{\prime}(z) d s ; \text { for } t \geq 0, z \in \mathbb{D} .
$$


Indeed,

$$
\begin{aligned}
G(z)\left(\left(f \circ \varphi_{t}\right)^{\prime}(z)-f^{\prime}(z)\right) & =f^{\prime}\left(\varphi_{t}(z)\right) G(z) \varphi_{t}^{\prime}(z)-m(z) \\
& =f^{\prime}\left(\varphi_{t}(z)\right) \frac{\partial \varphi_{t}(z)}{\partial t}-m(z) \\
& =m\left(\varphi_{t}(z)\right)-m(z)=\int_{0}^{t} \frac{\partial\left(m \circ \varphi_{s}\right)(z)}{\partial s} d s \\
& =\int_{0}^{t} G(z) m^{\prime}\left(\varphi_{s}(z)\right) \varphi_{s}^{\prime}(z) d s \\
& =G(z) \int_{0}^{t}\left(m \circ \varphi_{s}\right)^{\prime}(z) d s .
\end{aligned}
$$

Since $G$ is not identically null this verifies our assertion. Next let $I$ be an interval in $\partial \mathbb{D}$ and $R(I)$ the corresponding Carleson rectangle. For $0 \leq t \leq 1$ we have

$$
\begin{aligned}
\int_{R(I)}\left|\left(f \circ \varphi_{t}\right)^{\prime}(z)-f^{\prime}(z)\right|^{2}\left(1-|z|^{2}\right) d A(z) & =\int_{R(I)}\left|\int_{0}^{t}\left(m \circ \varphi_{s}\right)^{\prime}(z) d s\right|^{2}\left(1-|z|^{2}\right) d A(z) \\
& \leq \int_{R(I)} t\left(\int_{0}^{1}\left|\left(m \circ \varphi_{s}\right)^{\prime}(z)\right|^{2} d s\right)\left(1-|z|^{2}\right) d A(z)
\end{aligned}
$$

where we have applied Cauchy-Schwarz in the inside integral. Dividing by $|I|$, taking sup and interchanging the integrals we have

$$
\begin{aligned}
& \sup _{I \subseteq \partial \mathbb{D}}\left(\frac{1}{|I|} \int_{R(I)}\left|\left(f \circ \varphi_{t}\right)^{\prime}(z)-f^{\prime}(z)\right|^{2}\left(1-|z|^{2}\right) d A(z)\right)^{\frac{1}{2}} \\
& \leq \sup _{I \subseteq \partial \mathbb{D}}\left(\frac{1}{|I|} \int_{R(I)} t\left(\int_{0}^{1}\left|\left(m \circ \varphi_{s}\right)^{\prime}(z)\right|^{2} d s\right)\left(1-|z|^{2}\right) d A(z)\right)^{\frac{1}{2}} \\
& \leq \sup _{I \subseteq \partial \mathbb{D}}\left(t \int_{0}^{1}\left(\frac{1}{|I|} \int_{R(I)}\left|\left(m \circ \varphi_{s}\right)^{\prime}(z)\right|^{2}\left(1-|z|^{2}\right) d A(z)\right) d s\right)^{\frac{1}{2}} \\
& \leq\left(t \int_{0}^{1}\left\|m \circ \varphi_{s}\right\|_{B M O A}^{2} d s\right)^{\frac{1}{2}} \\
& \leq \sqrt{t} \sup _{s \in[0,1]}\left\|m \circ \varphi_{s}\right\|_{B M O A} \\
& \leq \sqrt{t} C\|m\|_{B M O A} \sup _{s \in[0,1]}\left(1-\log \left(1-\varphi_{s}(0)\right)\right. \\
& \leq C^{\prime} \sqrt{t}
\end{aligned}
$$

where $C^{\prime}>0$ is a certain constant not depending on $t$. Hence,

$$
\left\|C_{t} f-f\right\|_{B M O A} \leq\left|f\left(\varphi_{t}(0)\right)-f(0)\right|+C^{\prime} \sqrt{t} .
$$

Since $\lim _{t \rightarrow 0^{+}} \varphi_{t}(0)=0$ we find that $\lim _{t \rightarrow 0^{+}}\left\|C_{t} f-f\right\|_{B M O A}=0$, hence $f \in\left[\varphi_{t}, B M O A\right]$. We have shown $\left\{f \in B M O A: G f^{\prime} \in B M O A\right\} \subset\left[\varphi_{t}, B M O A\right]$, and the desired inclusion follows by taking closures.

If $\left(\varphi_{t}\right)$ is a semigroup of analytic functions then every composition operator $C_{t}(f)=f \circ \varphi_{t}$ is bounded on $V M O A$. This is because each $\varphi_{t}$ belongs to the Dirichlet space $\mathcal{D}$ (recall that $\varphi_{t}$ is univalent) and therefore also in $V M O A$. Thus the composition semigroup $\left(C_{t}\right)$ consists of bounded operators on $V M O A$.

Moreover $\left(C_{t}\right)$ is strongly continuous on $V M O A$ for every semigroup $\left(\varphi_{t}\right)$. This was stated in [16] without proof, and is contained as a special case among the $Q_{p, 0}$ spaces in [18, Theorem 4.1]. 
A short proof goes as follows. Strong continuity requires that $\lim _{t \rightarrow 0}\left\|f \circ \varphi_{t}-f\right\|_{B M O A}=0$ for each $f \in V M O A$. For a polynomial $P$ we can write

$$
\begin{aligned}
\left\|f \circ \varphi_{t}-f\right\|_{B M O A} & \leq\left\|f \circ \varphi_{t}-P \circ \varphi_{t}\right\|_{B M O A}+\left\|P \circ \varphi_{t}-P\right\|_{B M O A}+\|P-f\|_{B M O A} \\
& \leq\left(\left\|C_{t}\right\|_{V M O A}+1\right)\|P-f\|_{B M O A}+\left\|P \circ \varphi_{t}-P\right\|_{B M O A} .
\end{aligned}
$$

Since $V M O A$ contains the polynomials as a dense set and since $\sup _{0 \leq t<1}\left\|C_{t}\right\|_{V M O A}<\infty$, it suffices to show $\lim _{t \rightarrow 0}\left\|P \circ \varphi_{t}-P\right\|_{B M O A}=0$ for each polynomial. This now follows from the inequality $\|g\|_{B M O A} \leq\|g\|_{\mathcal{D}}$ between the $V M O A$-norm and the Dirichlet space norm which is valid for all $g \in \mathcal{D}$, see (2.1), along with the fact that every semigroup generates a semigroup of operators on the Dirichlet space [15, Theorem 1].

We proceed however to provide an alternative direct proof of this result which does not use [15, Theorem 1] and which is based on the $V M O A-H^{1}$ duality. Recall that this duality is realized by the pairing,

$$
\langle f, g\rangle:=\lim _{r \rightarrow 1} \int_{0}^{2 \pi} f\left(r e^{i \theta}\right) \overline{g\left(r e^{i \theta}\right)} \frac{d \theta}{2 \pi}, \quad f \in V M O A, g \in H^{1} .
$$

If we restrict the choice of $f$ and $g$, for example if both are chosen to lie in $H^{2}$, then the pairing can be expressed by the Littlewood-Paley identity (see [20, 8.1.9]):

$$
\langle f, g\rangle=f(0) \overline{g(0)}+2 \int_{\mathbb{D}} f^{\prime}(z) \overline{g^{\prime}(z)} \log \frac{1}{|z|} d A(z) .
$$

Now, we present another formulation of this dual pair involving functions in some other different spaces which will be more convenient for our purposes.

Lemma 2.3. If $f \in \mathcal{D}$ and $g \in H^{1}$, then

$$
\langle f, g\rangle=f(0) \overline{g(0)}+2 \int_{\mathbb{D}} f^{\prime}(z) \overline{g^{\prime}(z)} \log \frac{1}{|z|} d A(z) .
$$

Proof. Select a sequence $\left(g_{n}\right)$ in $H^{2}$ converging to $g$ in $H^{1}$. Using the Littewood-Paley identity, we have

and $\langle f, g\rangle=\lim _{n \rightarrow \infty}\left\langle f, g_{n}\right\rangle$.

$$
\left\langle f, g_{n}\right\rangle=f(0) \overline{g_{n}(0)}+2 \int_{\mathbb{D}} f^{\prime}(z) \overline{g_{n}^{\prime}(z)} \log \frac{1}{|z|} d A(z)
$$

Now,

$$
\begin{aligned}
& \int_{\mathbb{D}}\left|f^{\prime}(z)\left(g_{n}^{\prime}(z)-g^{\prime}(z)\right)\right| \log \frac{1}{|z|} d A(z) \leq \int_{|z|>1 / 2}\left|f^{\prime}(z)\left(g_{n}^{\prime}(z)-g^{\prime}(z)\right)\right| \log \frac{1}{|z|} d A(z) \\
+ & \int_{|z| \leq 1 / 2}\left|f^{\prime}(z)\left(g_{n}^{\prime}(z)-g^{\prime}(z)\right)\right| \log \frac{1}{|z|} d A(z) \\
\leq & C_{1}\left(\int_{\mathbb{D}}\left|f^{\prime}(z)\right|^{2} d A(z)\right)^{\frac{1}{2}}\left(\int_{\mathbb{D}}\left|g_{n}^{\prime}(z)-g^{\prime}(z)\right|^{2}(1-|z|)^{2} d A(z)\right)^{\frac{1}{2}} \\
+ & \int_{|z| \leq 1 / 2}\left|f^{\prime}(z)\left(g_{n}^{\prime}(z)-g^{\prime}(z)\right)\right| \log \frac{1}{|z|} d A(z)
\end{aligned}
$$

For any function $h=\sum_{n=0}^{\infty} a_{n} z^{n} \in H^{1}$, we obtain from Hardy's inequality (see [8, Theorem $6.2])$ that

$$
\int_{\mathbb{D}}\left|h^{\prime}(z)\right|^{2}(1-|z|)^{2} d A(z) \leq C_{2} \sum_{n=0}^{\infty} \frac{\left|a_{n}\right|^{2}}{n} \leq C_{2}\|h\|_{H^{1}}^{2}
$$

Hence,

$$
\int_{\mathbb{D}}\left|g_{n}^{\prime}(z)-g^{\prime}(z)\right|^{2}(1-|z|)^{2} d A(z) \leq C_{2}\left\|g_{n}-g\right\|_{H^{1}}^{2}
$$


Finally, applying that $f^{\prime}(z) \log \frac{1}{|z|} \in L^{1}(d A)$ and the Lebesgue's dominated convergence theorem, one also has

$$
\lim _{n \rightarrow \infty} \int_{|z| \leq 1 / 2}\left|f^{\prime}(z)\left(g_{n}^{\prime}(z)-g^{\prime}(z)\right)\right| \log \frac{1}{|z|} d A(z)=0 .
$$

Theorem 2.4. Every semigroup $\left(\varphi_{t}\right)$ generates a semigroup of operators on VMOA.

Proof. From the general theory of operator semigroups, a semigroup which is weakly continuous on a Banach space is in fact strongly continuous [17, p. 233]). Thus it suffices to prove that for each $f \in V M O A$ we have

$$
w-\lim _{t \rightarrow 0^{+}} C_{t}(f)=f
$$

where $w$ - denotes the weak limit. In other words for each fixed $f \in V M O A$ we want to prove

$$
\lim _{t \rightarrow 0^{+}}\left\langle C_{t}(f), g\right\rangle=\langle f, g\rangle
$$

for every $g \in H^{1}$. Arguing as before about the density of polynomials in $V M O A$ and the fact that $\sup _{0 \leq t<1}\left\|C_{t}\right\|_{V M O A}<\infty$ we see that it suffices to prove this for $f=P$ a polynomial.

Now, using again the Area Theorem ( $\varphi_{t}$ is univalent), we find

$$
\int_{\mathbb{D}}\left|P^{\prime}\left(\varphi_{t}(z)\right) \varphi_{t}^{\prime}(z)\right|^{2} d A(z) \leq \int_{\mathbb{D}}\left\|P^{\prime}\right\|_{\infty}^{2}\left|\varphi_{t}^{\prime}(z)\right|^{2} d A(z) \leq\left\|P^{\prime}\right\|_{\infty}^{2},
$$

so we can apply Lemma 2.3. Therefore, if $P$ a polynomial and $g \in H^{1}$ we deduce

$$
\left\langle P \circ \varphi_{t}-P, g\right\rangle=\left(P\left(\varphi_{t}(0)\right)-P(0)\right) g(0)+2 \int_{\mathbb{D}}\left(P \circ \varphi_{t}-P\right)^{\prime}(z) g^{\prime}(z) \log \frac{1}{|z|} \mid d A(z) .
$$

For each $\delta>0$, we split the integral

$$
\begin{gathered}
\int_{\mathbb{D}}\left|\left(P \circ \varphi_{t}-P\right)^{\prime}(z)\right|\left|g^{\prime}(z)\right| \log \frac{1}{|z|} d A(z) \leq \\
\left.\leq \int_{|z|>\delta} \mid\left(P \circ \varphi_{t}\right)-P\right)^{\prime}(z)|| g^{\prime}(z) \mid \log \frac{1}{|z|} d A(z) \\
+\int_{|z| \leq \delta}\left|\left(P \circ \varphi_{t}-P\right)^{\prime}(z)\right|\left|g^{\prime}(z)\right| \log \frac{1}{|z|} d A(z)=(1)+(2) .
\end{gathered}
$$

To estimate the first integral, we use that $\log \frac{1}{|z|} \approx 1-|z|$ and apply Cauchy-Schwarz's to obtain

$$
\begin{aligned}
(1) & \leq C\left\|P^{\prime}\right\|_{\infty} \int_{|z|>\delta}\left(\left|\varphi_{t}^{\prime}(z)\right|+1\right)\left|g^{\prime}(z)\right|(1-|z|) d A(z) \\
& \leq C\left\|P^{\prime}\right\|_{\infty}\left(\left(\int_{\mathbb{D}}\left|\varphi_{t}^{\prime}(z)\right|^{2} d A(z)\right)^{\frac{1}{2}}+1\right)\left(\int_{|z|>\delta}\left|g^{\prime}(z)\right|^{2}(1-|z|)^{2} d A(z)\right)^{\frac{1}{2}} \\
& \leq 2 C\left\|P^{\prime}\right\|_{\infty}\left(\int_{|z|>\delta}\left|g^{\prime}(z)\right|^{2}(1-|z|)^{2} d A(z)\right)^{\frac{1}{2}} .
\end{aligned}
$$

Now using (2.4) one has that $g^{\prime}(z)(1-|z|) \in L^{2}(d A)$, which shows that given $\varepsilon>0$ and, for all $t>0$, there exists $0<\delta_{0}<1$ such that

$$
\left.\int_{|z|>\delta_{0}} \mid\left(P \circ \varphi_{t}\right)-P\right)^{\prime}(z)|| g^{\prime}(z)\left|\log \frac{1}{|z|}\right| d A(z)<\varepsilon .
$$

At the same time, for every $z \in \mathbb{D}$,

$$
\lim _{t \rightarrow 0}\left(P \circ \varphi_{t}-P\right)^{\prime}(z)=0 .
$$


Therefore, using that $g^{\prime}(z) \log \frac{1}{|z|} \in L^{1}(d A)$ and the Lebesgue dominated convergence theorem, one concludes that

$$
\lim _{t \rightarrow 0} \int_{|z| \leq \delta_{0}}\left|\left(P\left(\varphi_{t}\right)-P\right)^{\prime}(z)\right|\left|g^{\prime}(z)\right| \log \frac{1}{|z|} d A(z)=0
$$

\section{VMOA and Maximal Subspaces}

This section is devoted to analyze those semigroups $\left(\varphi_{t}\right)$ such that $V M O A=\left[\varphi_{t}, B M O A\right]$. Since $V M O A$ is always contained in $\left[\varphi_{t}, B M O A\right]$ we see that $V M O A=\left[\varphi_{t}, B M O A\right]$ is equivalent to the following statement: if $f \in B M O A$, then

$$
f \in V M O A \text { if and only if } \lim _{t \rightarrow 0^{+}}\left\|f \circ \varphi_{t}-f\right\|_{B M O A}=0 .
$$

It is easy to see that, in general, $V M O A \varsubsetneqq\left[\varphi_{t}, B M O A\right]$. The easiest example of this type is the semigroup

$$
\varphi_{t}(z)=e^{-t} z+1-e^{-t}, \quad t \geq 0, z \in \mathbb{D} .
$$

For this semigroup the function $f(z)=\log (1-z) \in B M O A \backslash V M O A$ satisfies

$$
\left\|f \circ \varphi_{t}-f\right\|_{B M O A}=\left\|\log \left(e^{-t}(1-z)\right)-\log (1-z)\right\|_{B M O A}=2 \log e^{-t} \longrightarrow 0,
$$

thus $f \in\left[\varphi_{t}, B M O A\right]$. In fact it is easy to construct general examples of semigroups with this behavior. For instance take any starlike univalent function $h: \mathbb{D} \rightarrow \mathbb{C}$ with $h(0)=0$ and $h \in$ $B M O A \backslash V M O A$ and define $\varphi_{t}(z)=h^{-1}\left(e^{-t} h(z)\right)$. Then

$$
\left\|h \circ \varphi_{t}-h\right\|_{B M O A}=\left|e^{-t}-1\right|\|h\|_{B M O A} \longrightarrow 0,
$$

so that $h \in\left[\varphi_{t}, B M O A\right]$ while $h \notin V M O A$.

The following theorem gives a sufficient condition on the infinitesimal generator which assures that $V M O A=\left[\varphi_{t}, B M O A\right]$.

Theorem 3.1. Let $\left(\varphi_{t}\right)$ be a semigroup with infinitesimal generator $G$. Assume that for some $0<\alpha<1$,

$$
\frac{(1-|z|)^{\alpha}}{G(z)}=O(1) \text { as }|z| \rightarrow 1
$$

Then $V M O A=\left[\varphi_{t}, B M O A\right]$.

Proof. Since $\left[\varphi_{t}, B M O A\right]=\overline{\left\{f \in B M O A: G f^{\prime} \in B M O A\right\}}$, it suffices to show

$$
\left\{f \in B M O A: G f^{\prime} \in B M O A\right\} \subset V M O A .
$$

Let $g \in B M O A$ with $G g^{\prime} \in B M O A$. First we choose indices $p, p^{\prime}$ such that $1 / p+1 / p^{\prime}=1$, $2<p<\infty$, and such hat $\alpha<\frac{1}{p^{\prime}}<\alpha+\frac{1}{2}$. Hence $\alpha=\frac{1}{p^{\prime}}-\varepsilon$ with $0<\varepsilon<1 / 2$. We use the usual notation

$$
M_{p}(f, r)=\left(\int_{0}^{2 \pi}\left|f\left(r e^{i \theta}\right)\right|^{p} \frac{d \theta}{2 \pi}\right)^{1 / p}
$$

and we have, taking into account that $B M O A \subset H^{p}$, for $0<\delta \leq r<1$,

$$
M_{p}\left(g^{\prime}, r\right)=M_{p}\left(G g^{\prime} \frac{1}{G}, r\right) \leq M_{p}\left(G g^{\prime}, r\right) M_{\infty}\left(\frac{1}{G}, r\right) \leq \frac{C_{p}}{(1-r)^{1 / p^{\prime}-\varepsilon}} .
$$

In order to show that $g \in V M O A$ we will use the characterization of $V M O A$ in terms of Carleson measures (see [20, 8.2.5 and 8.4.2]) and it suffices prove

$$
\lim _{|z| \rightarrow 1} \int_{\mathbb{D}}\left|g^{\prime}(w)\right|^{2} \frac{\left(1-|w|^{2}\right)\left(1-|z|^{2}\right)}{|1-z \bar{w}|^{2}} d A(w)=0
$$


Now let $q=p / 2>1$ and apply Hölder's inequality for the pair of indices $q, q^{\prime}$ that is, $\frac{2}{p}+\frac{1}{q^{\prime}}=1$ to obtain

$$
\begin{aligned}
\int_{\mathbb{D}}\left|g^{\prime}(w)\right|^{2} & \frac{\left(1-|w|^{2}\right)\left(1-|z|^{2}\right)}{|1-z \bar{w}|^{2}} d A(w)=\int_{0}^{1} \int_{0}^{2 \pi}\left|g^{\prime}\left(r e^{i \theta}\right)\right|^{2} \frac{\left(1-r^{2}\right)\left(1-|z|^{2}\right)}{\left|1-z r e^{-i \theta}\right|^{2}} d \theta r d r \\
& \leq \int_{0}^{1} M_{p}^{2}\left(g^{\prime}, r\right)\left(\int_{0}^{2 \pi} \frac{\left(1-r^{2}\right)^{q^{\prime}}\left(1-|z|^{2}\right)^{q^{\prime}}}{\left|1-z r e^{-i \theta}\right|^{2 q^{\prime}}} d \theta\right)^{\frac{1}{q^{\prime}}} d r \\
& \leq C(1-|z|) \int_{0}^{1} M_{p}^{2}\left(g^{\prime}, r\right) \frac{(1-r)}{(1-|z| r)^{2-\frac{1}{q^{\prime}}}} d r=C(1-|z|) Q(|z|),
\end{aligned}
$$

where the last inequality follows from standard estimates on the Poisson kernel, see [8, p. 65], and $Q(|z|)$ denotes the last integral. We now have

$$
\begin{aligned}
Q(|z|) & =\int_{0}^{\delta} M_{p}^{2}\left(g^{\prime}, r\right) \frac{(1-r)}{(1-|z| r)^{2-\frac{1}{q^{\prime}}}} d r+\int_{\delta}^{1} M_{p}^{2}\left(g^{\prime}, r\right) \frac{(1-r)}{(1-|z| r)^{2-\frac{1}{q^{\prime}}}} d r \\
& \leq M_{p}^{2}\left(g^{\prime}, \delta\right) \int_{0}^{\delta} \frac{1}{(1-|z| r)^{\frac{1}{q}}} d r+C_{p}^{2} \int_{\delta}^{1} \frac{(1-r)^{1-2 / p^{\prime}+2 \varepsilon}}{(1-|z| r)^{2-\frac{1}{q^{\prime}}}} d r \\
& \leq C_{1} \int_{0}^{1} \frac{1}{(1-r)^{\frac{1}{q}}} d r+C_{2} \int_{0}^{1} \frac{(1-r)^{1-2 / p^{\prime}+2 \varepsilon}}{(1-|z| r)^{2-\frac{1}{q^{\prime}}}} d r \\
& =C_{1}^{\prime}+C_{2} \int_{0}^{1} \frac{(1-r)^{1-2 / p^{\prime}+2 \varepsilon}}{(1-|z| r)^{2-\frac{1}{q^{\prime}}}} d r \\
& \leq C_{1}^{\prime}+C_{2}^{\prime}(1-|z|)^{-1+2 \varepsilon}
\end{aligned}
$$

where the last integral was calculated by integration by parts as in [14, Lemma 6]. Putting all these together we find

$$
\begin{aligned}
\int_{\mathbb{D}}\left|g^{\prime}(w)\right|^{2} \frac{\left(1-|w|^{2}\right)\left(1-|z|^{2}\right)}{|1-z \bar{w}|^{2}} d A(w) & \leq C(1-|z|) Q(|z|) \\
& \leq C(1-|z|)\left(C_{1}^{\prime}+C_{2}^{\prime}(1-|z|)^{-1+2 \varepsilon}\right) \\
& \leq C^{\prime \prime} \max \left\{(1-|z|),(1-|z|)^{2 \varepsilon}\right\},
\end{aligned}
$$

and the proof is complete.

As an immediate corollary we have

Corollary 3.2. Suppose $\left(\varphi_{t}\right)$ is a semigroup whose infinitesimal generator $G$ satisfies condition (3.1) of Theorem 3.1. Then for a function $f \in B M O A$ the following are equivalent

(1) $f \in V M O A$.

(2) $\lim _{t \rightarrow 0^{+}}\left\|f \circ \varphi_{t}-f\right\|_{B M O A}=0$.

Clearly the semigroups $\varphi_{t}(z)=e^{-t} z$ and $\varphi_{t}(z)=e^{i t} z$ satisfy the condition (3.1) since, in both cases, the infinitesimal generator is $G(z)=c z$ for a certain nonzero constant $c$. Thus Theorem 3.1 gives an alternative proof (with entirely different techniques, see also [20]) of Sarason's result.

But there is a plethora of different semigroups $\left(\varphi_{t}\right)$ for which $V M O A=\left[\varphi_{t}, B M O A\right]$. A specific class of examples of this type are given by the semigroups associated with the generators

$$
G(z)=-z(1-z)^{\alpha}, \quad 0<\alpha<1 .
$$

To appreciate the breadth of the theorem recall that infinitesimal generators of semigroups with Denjoy-Wolff point $b=0$ have the form $G(z)=-z P(z)$ where $\operatorname{Re} P(z) \geq 0$. By Schwarz's lemma applied to $1 / P$ which also has nonnegative real part, $P(z)$ satisfies the growth condition

$$
\left|\frac{1}{P(z)}\right| \leq C_{2} \frac{1+|z|}{1-|z|} \quad \text { as }|z| \rightarrow 1 .
$$


Thus the most general infinitesimal generator of semigroups with inner Denjoy-Wolff point, fulfils the condition $\frac{1-|z|}{G(z)}=O(1)$.

Remark. Clearly, our $\mathrm{O}(1)$ condition is intimately related to the number and location of the zeros (in the angular sense) of the infinitesimal generator on the boundary of the unit disk. This topic has been partly analyzed in [6]. Another sufficient condition of the same nature which implies the conclusion of the theorem is

$$
\int_{|z| \geq \delta} \frac{d A(z)}{|G(z)|^{p}}<\infty
$$

for some $0<\delta<1$ and $p>2$. Indeed for $\delta<r<1$ we have

$$
(1-r) M_{p}^{p}(1 / G, r) \leq \int_{r}^{1} M_{p}^{p}(1 / G, s) d s,
$$

so by the finiteness of the integral we have

$$
M_{p}(1 / G, r)=o\left(\frac{1}{(1-r)^{\frac{1}{p}}}\right) .
$$

Now, using the Hardy-Littlewood estimates (see $[8,5.9])$ one has

$$
M_{\infty}(1 / G, r) \leq \frac{C M_{p}(1 / G, r)}{(1-r)^{\frac{1}{p}}}=o\left(\frac{1}{(1-r)^{\frac{2}{p}}}\right),
$$

and this little-oh condition is stronger than our big-Oh condition.

We now present a necessary condition for semigroups with inner Denjoy-Wolff for which $V M O A=$ $\left[\varphi_{t}, B M O A\right]$ holds. Observe that this necessary condition is quite close to the sufficient condition of Theorem 3.1.

Theorem 3.3. Let $\left(\varphi_{t}\right)$ be a semigroup with infinitesimal generator $G$ and Denjoy-Wolff point $b \in \mathbb{D}$. If $V M O A=\left[\varphi_{t}, B M O A\right]$ then

$$
\lim _{|z| \rightarrow 1} \frac{1-|z|}{G(z)}=0
$$

Proof. Without loss of generality, we may assume that $b=0$. The infinitesimal generator then is

$$
G(z)=-z P(z)
$$

where $P$ is analytic with $\operatorname{Re} P \geq 0$. If $P$ is constant, the result is clear. Otherwise consider the function

$$
m(z)=\int_{0}^{z} \frac{u}{G(u)} d u=-\int_{0}^{z} \frac{1}{P(u)} d u .
$$

Since $\operatorname{Re}(1 / P) \geq 0$ we deduce that $m \in B M O A$.

Now observe that

$$
\begin{aligned}
\left(m \circ \varphi_{t}\right)^{\prime}(z)-m^{\prime}(z) & =\frac{\varphi_{t}(z) \varphi_{t}^{\prime}(z)}{G\left(\varphi_{t}(z)\right)}-\frac{z}{G(z)}=\frac{z-\varphi_{t}(z)}{G(z)} \\
& =\frac{1}{G(z)} \int_{0}^{t} \frac{\partial \varphi_{s}(z)}{\partial s} d s=\int_{0}^{t} \varphi_{s}^{\prime}(z) d s
\end{aligned}
$$

where we have used (1.1) twice. Hence

$$
\left|\left(m \circ \varphi_{t}\right)^{\prime}(z)-m^{\prime}(z)\right|^{2}=\left|\int_{0}^{t} \varphi_{s}^{\prime}(z) d s\right|^{2} \leq t \int_{0}^{t}\left|\varphi_{s}^{\prime}(z)\right|^{2} d s .
$$


Now let $I \subset \partial \mathbb{D}$ be an interval and $R(I)$ the corresponding Carleson rectangle. We have

$$
\begin{aligned}
\frac{1}{|I|} \int_{R(I)}\left|\left(m \circ \varphi_{t}\right)^{\prime}(z)-m^{\prime}(z)\right|^{2} & \left(1-|z|^{2}\right) d A(z) \\
& \leq \frac{1}{|I|} \int_{R(I)}\left(t \int_{0}^{t}\left|\varphi_{s}^{\prime}(z)\right|^{2} d s\right)\left(1-|z|^{2}\right) d A(z) \\
& =t \int_{0}^{t} \frac{1}{|I|} \int_{R(I)}\left|\varphi_{s}^{\prime}(z)\right|^{2}\left(1-|z|^{2}\right) d A(z) d s \\
& \leq C t \int_{0}^{t} \int_{R(I)}\left|\varphi_{s}^{\prime}(z)\right|^{2} d A(z) d s \\
& \leq C t \int_{0}^{t} \int_{\mathbb{D}}\left|\varphi_{s}^{\prime}(z)\right|^{2} d A(z) d s \\
& =C t \int_{0}^{t}\left[\operatorname{Area}\left(\varphi_{s}(\mathbb{D})\right)\right]^{2} d s \\
& \leq C_{1} t^{2} .
\end{aligned}
$$

Now the norm $\left\|m \circ \varphi_{t}-m\right\|_{B M O A}$ equals

$$
\begin{aligned}
& \left|m\left(\varphi_{t}(0)\right)-m(0)\right|+\sup _{I \subset \partial \mathbb{D}}\left(\frac{1}{|I|} \int_{R(I)}\left|\left(m \circ \varphi_{t}\right)^{\prime}(z)-m^{\prime}(z)\right|^{2}\left(1-|z|^{2}\right) d A(z)\right)^{1 / 2} \\
& \leq\left|m\left(\varphi_{t}(0)\right)-m(0)\right|+C_{2} t
\end{aligned}
$$

so $\lim _{t \rightarrow 0}\left\|m \circ \varphi_{t}-m\right\|_{B M O A}=0$. Thus $m \in\left[\varphi_{t}, B M O A\right]$ and by the hypothesis $m \in V M O A$. The following standard argument for functions in $V M O A$ completes the proof. For $a \in \mathbb{D}$ write $\phi_{a}(z)=\frac{a-z}{1-\bar{a} z}$ then

$$
\begin{aligned}
\left(1-|a|^{2}\right)^{2}\left|m^{\prime}(a)\right|^{2} & =\left|\left(m \circ \phi_{a}\right)^{\prime}(0)\right|^{2} \leq\left\|m \circ \phi_{a}\right\|_{H^{2}}^{2} \\
& \leq C \int_{\mathbb{D}}\left|\left(m \circ \phi_{a}(z)\right)^{\prime}\right|^{2}\left(1-|z|^{2}\right) d A(z)
\end{aligned}
$$

(by the change of variables $w=\phi_{a}(z)$ )

$$
\begin{aligned}
& =C \int_{\mathbb{D}}\left|m^{\prime}(w)\right|^{2}\left(1-\left|\phi_{a}(w)\right|^{2}\right) d A(w) \\
& =C \int_{\mathbb{D}}\left|m^{\prime}(w)\right|^{2} \frac{\left(1-|a|^{2}\right)\left(1-|w|^{2}\right)}{|1-\bar{a} w|^{2}} d A(w)
\end{aligned}
$$

and this last integral tends to 0 as $|a| \rightarrow 1$ because $m \in V M O A$. It follows that

$$
\lim _{|a| \rightarrow 1} \frac{1-|a|}{G(a)}=\lim _{|a| \rightarrow 1} \frac{(1-|a|) m^{\prime}(a)}{a}=0 .
$$

We end this paper by characterizing those semigroups $\left(\varphi_{t}\right)$ of linear fractional maps such that $V M O A=\left[\varphi_{t}, B M O A\right]$. Roughly speaking, we show that, when dealing with semigroups of linear fractional maps, those found by Sarason are the unique where his theorem is true. For a detail analysis of these type of semigroups in one and several variables we refer the reader to [2].

Proposition 3.4. Let $\left(\varphi_{t}\right)$ be a semigroup such that each iterate is a linear fractional map. Then, $V M O A=\left[\varphi_{t}, B M O A\right]$ if and only if $\left(\varphi_{t}\right)$ has a fixed point in the unit disk but without fixed points in the boundary of the unit disk. 
Proof. We freely used some results from [2]. In particular, we use that the infinitesimal generator of a semigroup of linear fractional map is a polynomial of degree two.

Assume that $V M O A=\left[\varphi_{t}, B M O A\right]$. First of all, we are going to prove that the Denjoy-Wolff point of the semigroup $\left(\varphi_{t}\right)$ must be in the unit disk. Take $\sigma$ the Koenigs or univalent map of the semigroup (see [15]). It is known that $\sigma$ satisfies

$$
\sigma \circ \varphi_{t}=\sigma+t, \text { for all } t \geq 0
$$

If the semigroup is hyperbolic, using [5, Theorem 2.1], [9, page 283] and [11, page 78], we conclude that $\sigma \in B M O A \backslash V M O A$. Moreover, we observe that

$$
\left\|\sigma \circ \varphi_{t}-\sigma\right\|_{B M O A}=t \stackrel{t \rightarrow 0}{\longrightarrow} 0,
$$

and, therefore, $\sigma \in\left[\varphi_{t}, B M O A\right]$. Hence, the equality we are dealing with is impossible for this type of semigroups (we want to mention that this argument is indeed completely general, not only valid in the framework of semigroups of linear fractional maps).

If the semigroup is parabolic, then $\sigma$ is the Riemann map of a half-plane. In this case, taking $c \in \mathbb{C} \backslash \sigma(\mathbb{D})$, we see that $f(z)=\log (\sigma(z)-c) \in B M O A \backslash V M O A$ and, arguing as in the hyperbolic case, we obtain $f \in\left[\varphi_{t}, B M O A\right]$. Therefore, for this type of semigroups the equality is also impossible and we conclude that semigroup is necessarily elliptic (it has a fixed point in $\mathbb{D}$ ).

Therefore, we may assume in our proof that the semigroup has Denjoy-Wolff point point in $\mathbb{D}$. Now, since $V M O A=\left[\varphi_{t}, B M O A\right]$, using Theorem 3.3, we have

$$
\lim _{|z| \rightarrow 1} \frac{1-|z|}{G(z)}=0
$$

If the other fixed point is in the boundary, we have that the infinitesimal generator has the form

$$
G(z)=\lambda(z-a)(z-b), \text { with } a \in \partial \mathbb{D}, b \in \mathbb{D} \text { and } \lambda \neq 0 .
$$

Taking limits, we obtain a contradiction.

Likewise, assume now that $\left(\varphi_{t}\right)$ has no fixed point in the boundary of $\mathbb{D}$. In this case, the infinitesimal generator fits the following scheme:

$$
\begin{aligned}
G(z) & =\lambda(z-a)(z-b), \text { with } a \in \mathbb{C} \backslash \overline{\mathbb{D}}, b \in \mathbb{D} \text { and } \lambda \neq 0 \\
\text { or } G(z) & =\lambda(z-b), \text { with } b \in \mathbb{D} \text { and } \lambda \neq 0 .
\end{aligned}
$$

Finally, apply Theorem 3.1

\section{REFERENCES}

[1] E. Berkson and H. Porta, Semigroups of analytic functions and composition operators, Michigan Math. J. 25 (1978) 101-115.

[2] F. Bracci, M.D. Contreras, and S. Díaz-Madrigal, Infinitesimal generators associated with semigroups of linear fractional maps. J. Analyse Math. (to appear).

[3] P.S. Bourdon, J.A. Cima, and A.L. Matheson, Compact composition operators on BMOA, Trans. Amer. Math. Soc. 351 (1999) 2183-2196.

[4] M.D. Contreras and S. Díaz-Madrigal, Fractional iteration in the disk algebra: prime ends and composition operators, Revista Mat. Iberoamericana 21 (2005) 911-928.

[5] M.D. Contreras and S. Díaz-Madrigal, Analytic flows in the unit disk: angular derivatives and boundary fixed points, Pacific J. Math. 222 (2005) 253-286.

[6] M.D. Contreras, S. Díaz-Madrigal, and Ch. Pommerenke, On boundary critical points for semigroups of analytic functions, Math. Scand. 98 (2006) 125-142.

[7] N. Dunford and J. T. Schwartz, Linear operators, Part I. Wiley Classics Library, John Wiley \& Sons Inc., New York, 1988.

[8] P.L. Duren, Theory of $H^{p}$-spaces, Academic Press, New York, 1970.

[9] J.B. Garnett, Bounded Analytic Functions, Pure and Applied Mathematics, Vol. 96, Academic Press, New York, 1981.

[10] Ch. Pommerenke, Über die subordination analytischer Funktionen, J. Reine Angew. Math. 218 (1965) 159-173.

[11] Ch. Pommerenke, Boundary Behaviour of Conformal Maps, Springer-Verlag, Berlin, 1992.

[12] D. Sarason, Function of vanishing mean oscillation, Trans. Amer. Math. Soc. 207 (1975) 391-405. 
[13] D. Sarason, Function Theory on the Unit Circle, Virginia Poly. Inst. and State Univ. Blacksburg, Virginia, 1979.

[14] A. L. Shields and D. L. Williams, Bounded projections, duality and multipliers in spaces of analytic functions, Trans. Amer. Math. Soc. 162 (1971), 287-302.

[15] A. G. Siskakis, Semigroups of composition operators on the Dirichlet space, Results Math. 30 (1996), 165-173.

[16] A. G. Siskakis, Semigroups of composition operators on spaces of analytic functions, a review, Contemp. Math. 213 (1998) 229-252.

[17] K. Yosida, Functional Analysis, Springer-Verlag, Sixth Edition, Fundamental Principles of Mathematical Sciences, vol. 123, Springer-Verlag, Berlin-New York, 1980.

[18] K. J. Wirths and J. Xiao, Recognizing $Q_{p, 0}$ functions as per Dirichlet space structure, Bull. Belg. Math. Soc. $8(2001), 47-59$.

[19] J. Xiao, Holomorphic $Q$ Classes, Lecture Notes in Math. 1767, Springer-Verlag 2001.

[20] K. Zhu, Operator Theory in Function spaces, Marcel Dekker, Inc., New York and Basel, 1990.

Departamento de Análisis Matemático, Universidad de Valencia, 46100 Burjassot, Valencia, Spain.

E-mail address: Oscar.Blasco@uv.es

Camino de los Descubrimientos, s/n, Departamento de Matemática Aplicada II, Escuela Técnica SuPerior de Ingenieros, Universidad de Sevilla, 41092, Sevilla, Spain.

E-mail address: contreras@us.es

Camino de los Descubrimientos, s/n, Departamento de Matemática Aplicada it, Escuela Técnica Superior de Ingenieros, Universidad de Sevilla, 41092, Sevilla, Spain.

E-mail address: madrigal@us.es

Departamento de Análisis Matemático, Universidad de Valencia, 46100 Burjassot, Valencia, Spain. E-mail address: Josep.Martinez@uv.es

Department of Mathematics, Aristotle University of Thessaloniki, 54006 Thessaloniki, Greece.

E-mail address: siskakis@math.auth.gr 